\title{
Cardioprotective potential of Dendrobium officinale Kimura et Migo against myocardial ischemia in mice
}

\author{
MENG-MENG DOU ${ }^{1}$, ZHI-HAO ZHANG ${ }^{1}$, ZHU-BO LI $^{1}$, JIE ZHANG $^{2}$ and XIAO-YAN ZHAO ${ }^{1}$ \\ ${ }^{1}$ Department of Pharmacology, College of Pharmaceutical Sciences, Southwest University, Chongqing 400716; \\ ${ }^{2}$ Department of Neurology, The Ninth People's Hospital of Chongqing, Chongqing 400700, P.R. China
}

Received October 2, 2015; Accepted September 14, 2016

DOI: $10.3892 / \mathrm{mmr} .2016 .5789$

\begin{abstract}
Dendrobium officinale Kimura et Migo has been used for thousands of years to promote body fluid production; however, little is currently known regarding its effects on the heart. The present study aimed to explore the cardioprotective potential of the water extract of Dendrobium officinale Kimura et Migo (DOE) on myocardial ischemia in mice. A mouse model of myocardial ischemia was induced following ligation of the left anterior descending coronary artery. Prior to the operation, mice were administered a vehicle or DOE for 2 weeks. Following the operation, ST elevation was measured. To estimate the extent of myocardial damage, infarct size analysis and histopathological examination were performed. The activities of cardiac marker enzymes [creatine kinase (CK)-MB and lactate dehydrogenase (LDH)] and antioxidative indicators [malondialdehyde (MDA) and superoxide dismutase (SOD)] were also analyzed to explore the underlying mechanisms. Treatment with DOE decreased infarct size and the number of apoptotic cardiomyocytes; reduced serum CK-MB, $\mathrm{LDH}$ and MDA activities; and increased SOD levels. According to western blotting, DOE conferred protection against myocardial ischemic injury via the regulation of Meis1 expression. These results indicated that DOE may exert potential cardioprotective effects against myocardial ischemia; these effects may be associated with its antioxidant activity, and its ability to inhibit cardiac cell apoptosis and to regulate Meis1 expression.
\end{abstract}

Correspondence to: Dr Xiao-Yan Zhao, Department of Pharmacology, College of Pharmaceutical Sciences, Southwest University, 2 Tiansheng Road, Chongqing 400716, P.R. China E-mail: zhaoxiaoyan67@163.com

Dr Jie Zhang, Department of Neurology, The Ninth People's Hospital of Chongqing, Hejia Road, Chongqing 400700, P.R. China E-mail: zhjzhj121@163.com

Key words: Dendrobium officinale Kimura et Migo, myocardial ischemia, histopathological examination, apoptosis, Meis1

\section{Introduction}

Cardiovascular diseases (CVDs), particularly ischemic heart disease, are the primary cause of disability and mortality worldwide. The World Health Organization estimated that 17.5 million people succumbed to CVDs in 2012, and 7.4 million deaths were attributed to ischemic heart disease (1). The pathogenesis of myocardial ischemia, which is caused by atherosclerotic plaques or coronary artery occlusion (2), is considered to be multifactorial, and is associated with oxidative stress (3), mitochondrial dysfunction (4) and apoptotic cascade activation (5). Conversely, antioxidants may decrease cellular injury and apoptosis in ischemic hearts through radical-scavenging activities (6).

Natural products-based therapeutic strategies have been suggested as a potential option for the treatment of patients with myocardial ischemia (7). Dendrobium officinale Kimura et Migo (Orchidaceae family) is a prized traditional Chinese medicine, which has been used clinically to promote body fluid production and maintain gastric tonicity in China and Southeast Asia (8). Dendrobium officinale has been reported to possess diverse pharmacological properties, including anti-inflammatory (9), immunomodulatory (10), neuroprotective (11) and antitumor (12) activities. Dendrobium officinale polysaccharides, which are a potential candidate for the treatment of Sjögren's syndrome (13), were able to suppress the overexpression of proinflammatory cytokines, including tumor necrosis factor- $\alpha$, interleukin (IL)-1 $\beta$ and IL-6, in a mouse model of Sjögren's syndrome, and inhibited apoptosis by downregulating the expression of caspase-3 and decreasing the B-cell lymphoma 2 (Bcl-2)-associated X protein/Bcl-2 ratio (9). Dendrobium officinale polysaccharides also possess immunomodulatory activity in vivo and in vitro, which is mediated by nitric oxide (10). Furthermore, dendrocandins extracted from Dendrobium officinale have been reported to promote neurite outgrowth in PC12 cells (14). Considering the antiaggregation activity of other Dendrobium species (15), the present study hypothesized that the water extract of Dendrobium officinale (DOE) may exert cardioprotective effects against myocardial ischemia.

The homeodomain protein Meis1 is a three amino acid loop extension transcription factor, which participates in various physiological processes, including growth, proliferation and prevention of the accumulation of reactive oxygen 
species (16). Specifically, the Meis1/pre-B-cell leukemia homeobox 1/homeobox A9 complex is able to activate oncogenic genes, resulting in cell hyperproliferation (17). Meis1 is also required to establish definitive hematopoiesis in the mouse embryo, and its deletion in vivo may lead to agenesis of the megakaryocyte lineage and localized defects in vascular patterning (18). In terms of normal cardiac development, Meis1 acts as a regulator of cardiomyocyte cell cycle arrest and a potential transcriptional regulator of neonatal heart regeneration (19).

The present study aimed to evaluate the cardioprotective effects of DOE in vivo. Briefly, myocardial ischemia was induced in mice following ligation of the left anterior descending coronary artery (LAD). Subsequently, the effects of water-soluble components of Dendrobium officinale were investigated on myocardial injury, and the potential underlying mechanisms against cardiomyopathy were evaluated. The results suggested that pretreatment with DOE significantly decreased infarct size; reduced creatine kinase (CK)-MB and lactate dehydrogenase (LDH) levels in serum; and attenuated cardiac oxidative damage.

\section{Materials and methods}

Chemicals and materials. CK-MB (cat. no. H197), LDH (cat. no. A020-1), total superoxide dismutase (SOD; cat. no. A001-1) and malondialdehyde (MDA; cat. no. A003-1) diagnostic agents were obtained from Nanjing Jiancheng Bioengineering Research Institute (Nanjing, China). Meis1 antibody (cat. no. BS3488) was obtained from Bioworld Technology, Co., Ltd. (Nanjing, China). GAPDH polyclonal primary antibody (cat. no. 10494-1-AP) and goat anti-rabbit horseradish peroxidase-conjugated immunoglobulin G secondary antibody (cat. no. SA00001-2) were purchased from Wuhan Sanying Biotechnology (Wuhan, China). All other reagents used were of commercial analytical grade.

DOE preparation. The dry stems of Dendrobium officinale were purchased from Xi'an Xiaocao Botanical Development Co. Ltd. (Xi'an, China). The voucher specimens were deposited at Southwest University (Chongqing, China). Briefly, the dry stems were ground into a fine powder through a 100-mesh filter. The powdered materials $(100 \mathrm{~g})$ were pre-extracted using petroleum ether and the residues were then extracted three times using hot distilled water. The crude extracts were filtered through a $0.22 \mu \mathrm{m}$ microporous membrane, concentrated via the alcohol precipitation method and centrifuged at $625 \mathrm{x} g$ for $10 \mathrm{~min}$. The extracts were then dried by lyophilization, and $23.5 \mathrm{~g}$ powder extracts were produced. The DOE predominantly consisted of polysaccharide, and the concentration of polysaccharide was determined by the phenol-sulfuric acid method to be $97.4 \%$.

Experimental animals. A total of 60 Kunming male mice (age, 4 weeks; weight, $22 \pm 2 \mathrm{~g}$ ) were purchased from Chongqing Tengxin Bio-technology Co., Ltd. (Chongqing, China). The present study was carried out according to recommendations of the National Institutes of Health Guide for the Care and Use of Laboratory Animals (2015). In addition, the experiments were approved by the Ethical Committee for Animals of Southwest University. The mice were housed in standard conditions:
Humidity, $50 \%$; temperature, $25 \pm 2^{\circ} \mathrm{C}$, under a $12: 12 \mathrm{~h}$ light/dark cycle, with ad libitum access to normal food and tap water. Mice were allowed to acclimate to the environment for 1 week prior to experimentation.

Experimental procedure. Mice were randomly assigned to five groups ( $\mathrm{n}=10 /$ group): Sham group, model group, and LAD + DOE pretreatment groups $(75,150$ and $300 \mathrm{mg} / \mathrm{kg}$ DOE; oral administration). DOE was dissolved in distilled water. Mice were administered normal saline $(10 \mathrm{ml} / \mathrm{kg})$ or DOE $(75,150$ or $300 \mathrm{mg} / \mathrm{kg}$ ) intragastrically once daily for 2 weeks. Subsequently, the mice were subjected to coronary artery ligation; LAD occlusion was performed as previously reported $(20,21)$. Briefly, the left chest cavity of the mice was opened and the heart was exposed following anesthetization with sodium pentobarbital ( $40 \mathrm{mg} / \mathrm{kg}$, i.p.). Subsequently, in the model and LAD + DOE pretreatment groups, 7-0 silk was used to ligate the LAD for $24 \mathrm{~h}$, after which the heart was returned to its normal position and the thoracic cavity was closed. In the sham group, 7-0 silk was passed through the LAD, without ligation. After $24 \mathrm{~h}$, mice were sacrificed by an overdose of pentobarbital sodium $(100 \mathrm{mg} / \mathrm{kg})$, and blood and heart samples were collected.

Electrocardiogram (ECG) record. The lead II ECG was recorded using the BL-420F biological function experiment system (Chengdu Taimeng Technology Co. Ltd., Chengdu, China). Significant ECG changes, including elevation of the ST segment and widening of the QRS complex, indicated successful coronary occlusion.

Determination of infarct size. A total of $24 \mathrm{~h}$ after ischemia, the hearts were collected and dissected at $1.2 \mathrm{~mm}$ cross-section. Subsequently, the heart sections were stained with $1 \%$ triphenyl tetrazolium chloride (TTC) for $15 \mathrm{~min}$ at $37^{\circ} \mathrm{C}$ in $0.2 \mathrm{M}$ phosphate-buffered saline (22). The viable myocardium was stained red, whereas the infarcted tissue remained pale. Images were captured and the infarct size was analyzed using Image-Pro Plus 6.0 software (Media Cybernetics, Inc., Rockville, MD, USA).

Determination of serum $C K-M B, L D H, S O D$ and MDA activity. Blood samples were collected and were centrifuged at $625 \mathrm{x} g$ for $10 \mathrm{~min}$ at $4^{\circ} \mathrm{C}$ to obtain serum samples. The CK-MB, LDH, SOD and MDA activity levels in the serum samples were determined using commercially available kits according to the manufacturer's protocols.

Histopathological examination. Heart tissues harvested from the mice were washed immediately with ice-cold saline. The biopsies were then fixed in $4 \%$ neutral paraformaldehyde and were embedded in paraffin. Heart tissues were cut into $5 \mu \mathrm{m}$ sections, which were stained with hematoxylin and eosin (H\&E) for $90 \mathrm{~min}$ at room temperature. Morphological evaluation and observation were performed under a light microscope.

Terminal deoxynucleotidyl transferase mediated dUTP nick end labeling (TUNEL) assay. TUNEL assays were performed as previously reported (23). The total number of TUNEL-positive cardiomyocyte nuclei in the infarcted zone was counted. 
A

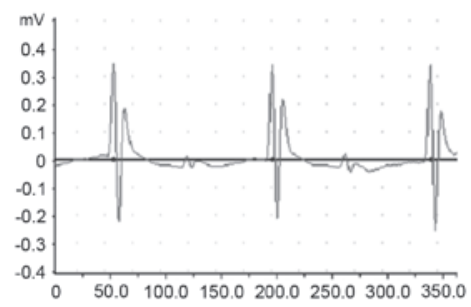

C

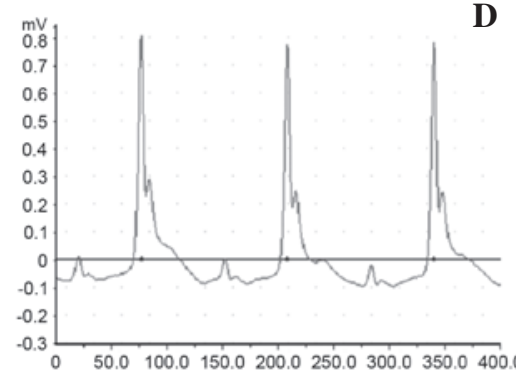

B
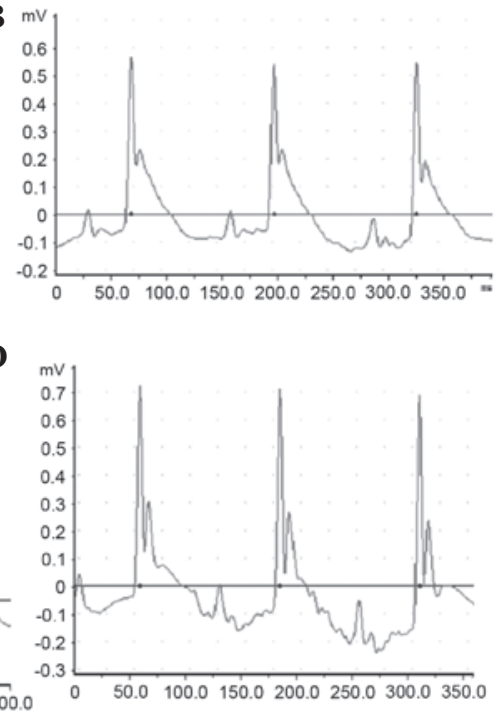

$\mathbf{E}$

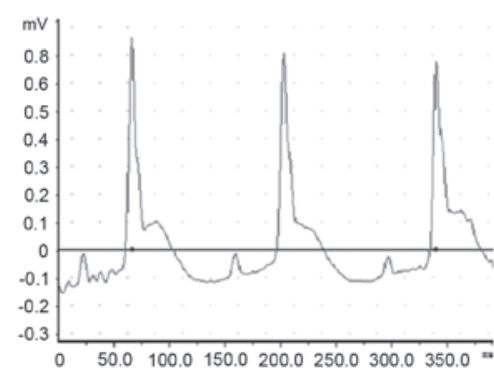

$\mathbf{F}$

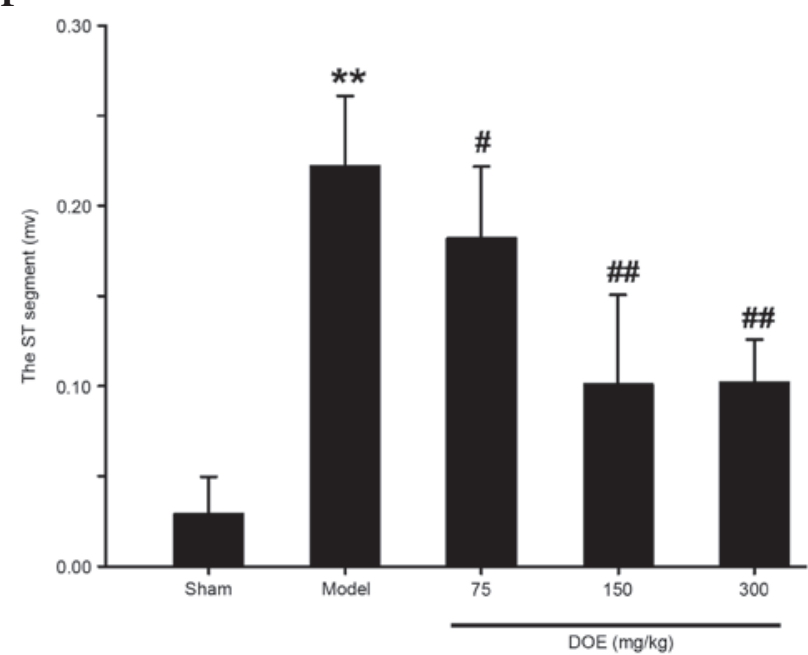

Figure 1. ECG recording in each group. The lead II ECG was recorded using a BL-420F biological function experiment system. (A) Sham group. (B) Model group. (C) DOE $75 \mathrm{mg} / \mathrm{kg}$ group. (D) DOE $150 \mathrm{mg} / \mathrm{kg}$ group. (E) DOE $300 \mathrm{mg} / \mathrm{kg}$ group. (F) ST segment changes in the ECG. Data are presented as the

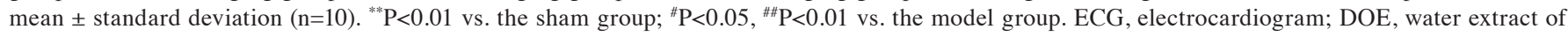
Dendrobium officinale.

Individual nuclei were visualized at a magnification of 400x (Olympus CKX41; Olympus Corporation, Tokyo, Japan), and the percentage of apoptotic nuclei (apoptotic nuclei/total nuclei) was calculated in six randomly selected fields per section and averaged for statistical analysis.

Western blotting. Heart tissues were lysed in radioimmunoprecipitation assay buffer [50 mM Tris $(\mathrm{pH} 7.4)$, $150 \mathrm{mM} \mathrm{NaCl}, 1 \%$ Triton X-100, $1 \%$ sodium deoxycholate, $1 \%$ SDS, $1 \mathrm{mM}$ phenylmethylsulfonyl fluoride], and concentration was determined using the bicinchoninic acid method. Heart tissue lysates $(100 \mu \mathrm{g})$ were separated by $12 \%$ SDS-PAGE, and the proteins were electrophoretically transferred to polyvinylidene difluoride membranes. After blocking non-specific binding sites for $2 \mathrm{~h}$ with $5 \%$ dried skim milk at room temperature, the membranes were individually exposed to primary antibodies (anti-Meis1, 1:6,000; anti-GAPDH, $1: 10,000)$ at $4^{\circ} \mathrm{C}$ overnight. The membranes were subsequently incubated for $2 \mathrm{~h}$ at room temperature with HRP-conjugated secondary anti-rabbit antibodies. The immunoreactive proteins were detected using an enhanced chemiluminescence reagent (Advansta Inc., Menlo Park, CA, USA). The proteins 
A

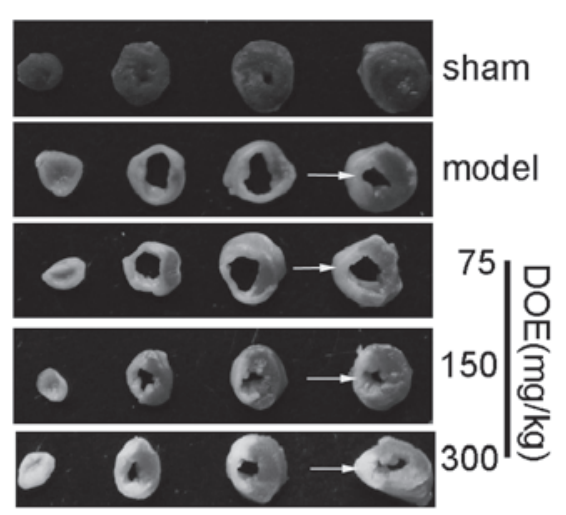

B

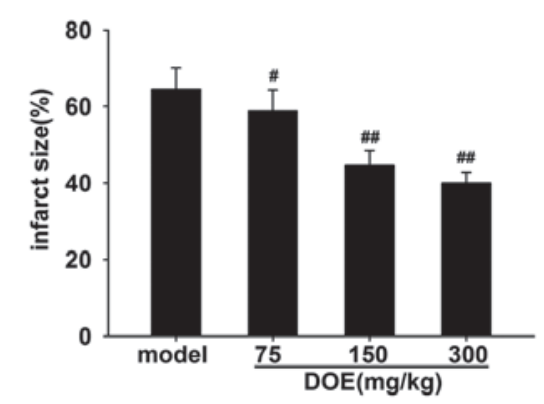

Figure 2. Effects of DOE on infarct size in the experimental mice. (A) Heart sections were stained with triphenyl tetrazolium chloride. Dark black areas (red staining; arrows) represent normal tissue and unstained pale areas indicate infarcted tissue. (B) Infarct size as a percentage of total volume. Data are presented as the mean \pm standard deviation $(\mathrm{n}=6) .{ }^{\#} \mathrm{P}<0.05,{ }^{\# \prime} \mathrm{P}<0.01$ vs. the model group. DOE, water extract of Dendrobium officinale.

were visualized and representative images were obtained. Densitometric analysis was performed using Quantity One 4.6.2 software (Bio-Rad Laboratories, Inc., Hercules, CA, USA) and GAPDH expression was used as an internal standard.

Statistical analysis. All statistical analyses were performed using SPSS statistical software (version 16.0 for Windows; SPSS Inc., Chicago, IL, USA). Data are presented as the mean \pm standard deviation. Statistical comparisons were performed using one-way analysis of variance followed by Tukey's post-hoc test for multiple group comparisons. $\mathrm{P}<0.05$ was considered to indicate a statistically significant difference.

\section{Results}

Effects of DOE on ECG parameters. The Lead II ECG is presented in Fig. 1. Marked elevation of the ST segment was detected in the myocardial ischemia model group, whereas a normal ECG was observed in the sham group. Ischemia-induced alterations to the ECG were significantly ameliorated by pretreatment with DOE, as compared with in the model group.

Effects of DOE on infarct size. To evaluate infarct size, TTC staining was performed. The cross-section of the heart stained with TTC is presented in Fig. 2A. As shown in Fig. 2B, DOE markedly decreased infarct size compared with the model group.
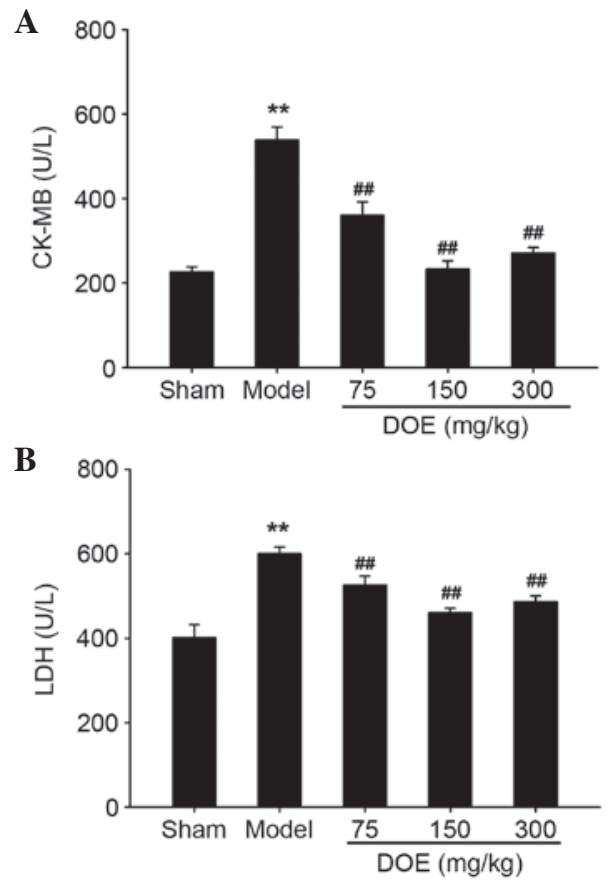

Figure 3. Effects of DOE administration on (A) CK-MB and (B) LDH levels in the serum. Data are presented as the mean \pm standard deviation $(\mathrm{n}=10) .{ }^{* *} \mathrm{P}<0.01$ vs. the sham group; ${ }^{\# \#} \mathrm{P}<0.01$ vs. the model group. CK-MB, creatine kinase-MB; LDH, lactate dehydrogenase; DOE, water extract of Dendrobium officinale.
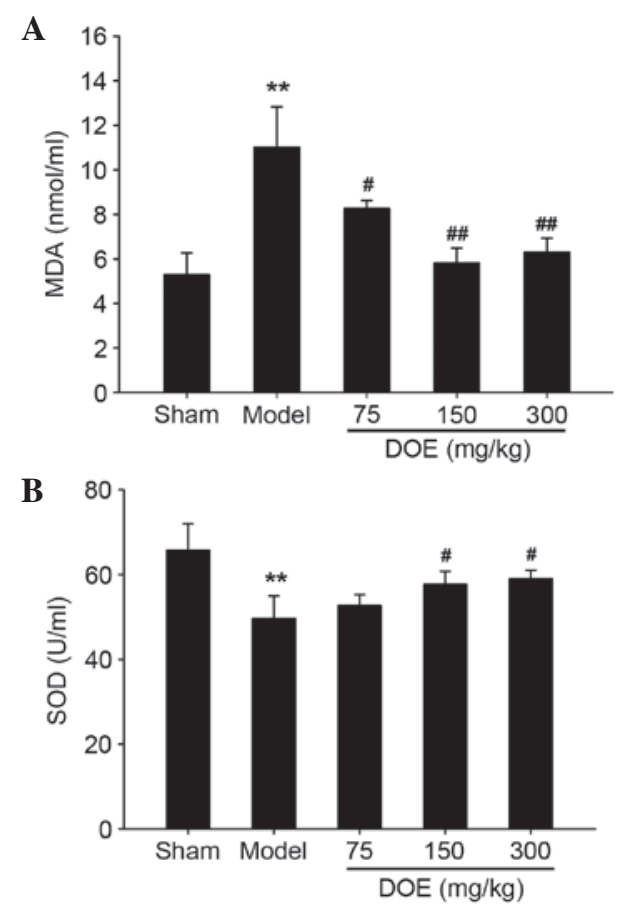

Figure 4. Effects of DOE administration on (A) MDA and (B) SOD levels in the serum. Data are presented as the mean \pm standard deviation $(\mathrm{n}=10) .{ }^{* *} \mathrm{P}<0.01$ vs. the sham group; ${ }^{\# P} \mathrm{P}<0.05,{ }^{\# \#} \mathrm{P}<0.01$ vs. the model group. MDA, malondialdehyde; SOD, superoxide dismutase; DOE, water extract of Dendrobium officinale.

A modest reduction in infarct size was detected in mice treated with $75 \mathrm{mg} / \mathrm{kg}$ DOE (5.6\% decrease). Notably, pretreatment with $150 \mathrm{mg} / \mathrm{kg}$ DOE more significantly reduced infarct size (10.0\% decrease). 


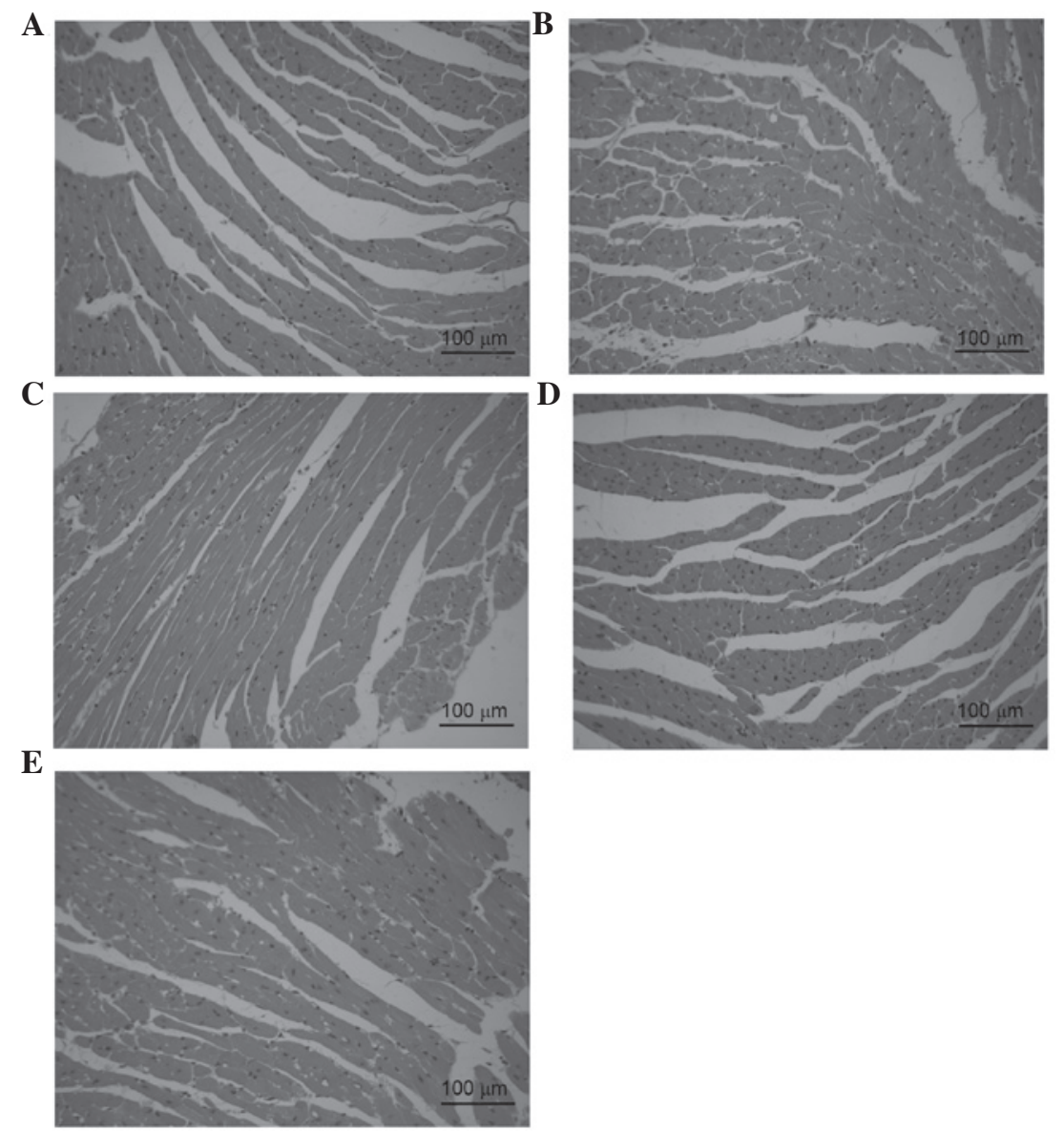

Figure 5. Effects of DOE administration on histopathological alterations in myocardial tissue. Mice were treated with various concentrations of DOE for 2 weeks prior to left anterior descending coronary artery ligation. Cardiac tissue then underwent hematoxylin and eosin staining. Representative images were captured at x200 magnification. (A) Sham group. (B) Model group. (C) DOE $75 \mathrm{mg} / \mathrm{kg}$ group. (D) DOE $150 \mathrm{mg} / \mathrm{kg}$ group. (E) DOE $300 \mathrm{mg} / \mathrm{kg}$ group. (Scale bar $=100 \mu \mathrm{m})$. DOE, water extract of Dendrobium officinale.

Effects of DOE on serum $C K-M B$ and $L D H$ levels. As shown in Fig. 3, CK-MB and LDH activities, which are indicators of myocardial ischemia, were significantly increased in the myocardial ischemia model group compared with the sham group. Conversely, pretreatment with DOE for 2 weeks decreased myocardial CK-MB and LDH release. Furthermore, the most obvious reduction was exhibited in mice pretreated with the middle dose of DOE $(150 \mathrm{mg} / \mathrm{kg})$. However, there was no marked difference in the alterations in CK-MB and LDH levels between the middle dose group $(150 \mathrm{mg} / \mathrm{kg})$ and the high dose group $(300 \mathrm{mg} / \mathrm{kg})$.

Effects of DOE on serum MDA and SOD levels. Myocardial ischemia is associated with oxidative stress (24); therefore, MDA and SOD levels were measured in the present study. As shown in Fig. 4, MDA activity was markedly increased in the model group, whereas pretreatment with DOE for 2 weeks decreased serum MDA levels. Furthermore, the most obvious decrease was observed in the middle dose group $(150 \mathrm{mg} / \mathrm{kg})$. Conversely, SOD activity was reduced in the model group compared with the sham group, whereas SOD levels were increased by pretreatment with DOE, as compared with in the model group. However, no significant change was detected in serum SOD levels between the low dose DOE group $(75 \mathrm{mg} / \mathrm{kg})$ and the model group.
Effects of DOE on histopathological myocardial alterations. Histopathological evaluation of cardiac tissue was conducted using H\&E staining, as presented in Fig. 5. The cardiac tissue in the sham group exhibited a clear structure without infiltration of inflammatory cardiomyocytes (Fig. 5A). However, myocardial structural abnormalities, including cytoplasmic vacuolization, cardiomyocyte necrosis and inflammatory infiltration, were detected in the model group (Fig. 5B). Conversely, the presence of necrotic cardiomyocytes was rare, and vacuolization and myofibrillar loss were almost undetectable following pretreatment with DOE (Fig. 5C-E).

Effects of DOE on myocardial apoptosis. Apoptotic alterations in cardiac tissue and the percentage of TUNEL-positive cells are presented in Fig. 6. As shown in Fig. 6A, TUNEL-positive cells were rarely detected in the sham group. However, the number of TUNEL-positive cells was markedly increased in the myocardial ischemia model group (Fig. 6B). The number of TUNEL-positive cells was significantly decreased $(42.5 \pm 12.4$, $37.3 \pm 1.6$ and $18.2 \pm 4.5 \%)$ by pretreatment with DOE $(75,150$ and $300 \mathrm{mg} / \mathrm{kg}$ ) compared with the model group $(54.2 \pm 7.5 \%)$.

Effects of DOE on the expression of Meis1. To determine the underlying mechanism of DOE, the protein expression levels of Meis1 were assessed by western blotting. The 
$\mathbf{A}$

C

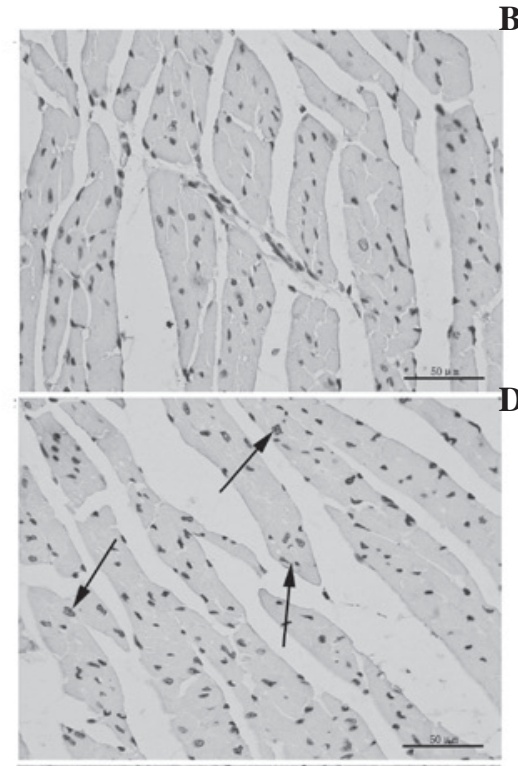

$\mathbf{E}$

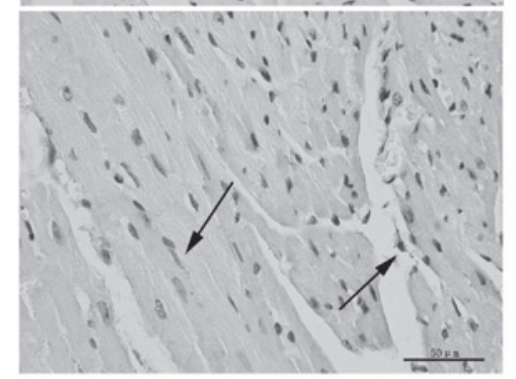

B
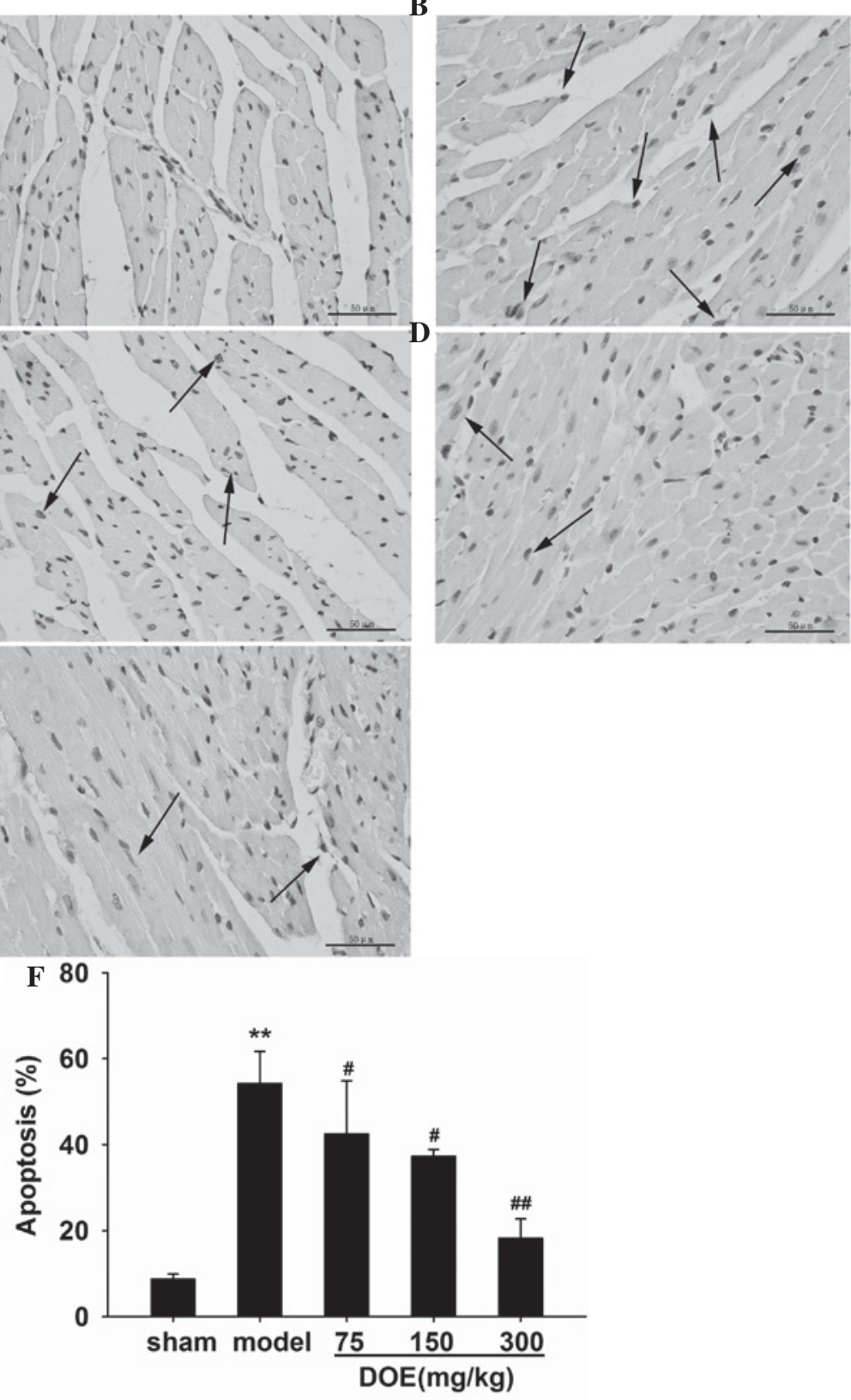

Figure 6. Effects of DOE on apoptosis induced by left anterior descending coronary artery ligation (scale bar=50 $\mu \mathrm{m}$; original magnification $\mathrm{x} 400$ ). Cardiac tissue was processed with the TUNEL assay. (A) Sham group. (B) Model group. (C) DOE $75 \mathrm{mg} / \mathrm{kg}$ group. (D) DOE $150 \mathrm{mg} / \mathrm{kg}$ group. (E) DOE $300 \mathrm{mg} / \mathrm{kg}$ group. TUNEL-positive cells are indicated with arrows. (F) TUNEL-positive cell percentage. Data are presented as the mean \pm standard deviation ( $\mathrm{n}=6$ ). ** $\mathrm{P}<0.01 \mathrm{vs}$. the sham group; ${ }^{*} \mathrm{P}<0.05,{ }^{\# \#} \mathrm{P}<0.01$ vs. the model group. DOE, water extract of Dendrobium officinale; TUNEL, terminal deoxynucleotidyl transferase mediated dUTP nick end labeling.

results presented in Fig. 7 indicate that the expression levels of Meis1 were significantly decreased in the model group compared with the sham group. Conversely, pretreatment with DOE (75 and $150 \mathrm{mg} / \mathrm{kg}$ ) markedly upregulated the expression of Meis1.

\section{Discussion}

The present study demonstrated that pretreatment with DOE significantly inhibited ST segment elevation, reduced infarct size and attenuated cardiomyocyte apoptosis. Furthermore, DOE prevented CK-MB and LDH release, decreased serum MDA levels, increased serum SOD levels and upregulated the protein expression levels of Meis1. These results suggested that DOE may serve a protective role against ischemic cardiomyopathy, and may be considered a potential clinical agent in the attenuation and prevention of ischemic injury.

ECG is an important clinical tool used to evaluate heart function. ST segment monitoring is sensitive during the development of ischemia (25). ST segment elevation, which is a reliable biomarker for the diagnosis of myocardial ischemia, is associated with continuous damage to the cell membrane (26). In the present study, ST segment elevation was observed in the model group; however, ST segment elevation was alleviated by pretreatment with DOE. These data indicated that DOE may ameliorate cardiac ischemia. 


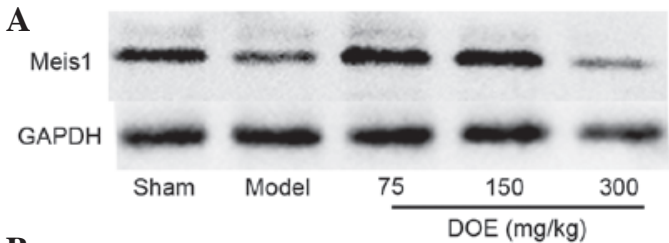

B

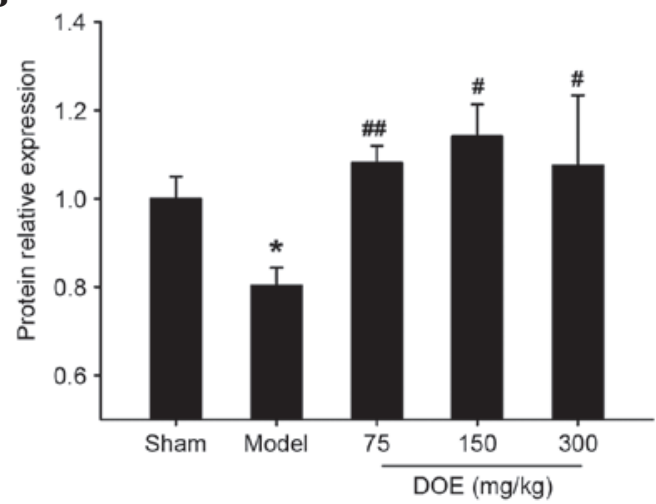

Figure 7. Effects of DOE on Meis1 expression in cardiac tissue. (A) The protein expression levels of Meis1 were assessed using western blotting. Representative immunoblots are presented. GAPDH was used as an internal control. (B) Blots were semi-quantified. Data are presented as the mean \pm standard deviation $(\mathrm{n}=6) .{ }^{*} \mathrm{P}<0.05$ vs. the sham group; ${ }^{*} \mathrm{P}<0.05$, ${ }^{\# \#} \mathrm{P}<0.01$ vs. the model group. DOE, water extract of Dendrobium officinale.

TTC staining was used to evaluate infarct size. TTC is considered a proton acceptor for pyridine nucleotide-linked-dehydrogenases together with cytochromes, which comprise an integral part of the inner mitochondrial membrane and form the electron transport chain (27). Tetrazolium salt is reduced by these enzymes into lipid formazan in cardiac tissue, resulting in red staining. Therefore, viable tissue is stained red, whereas infarcted tissue is stained white. The present study demonstrated that DOE administration significantly reduced LAD ligation-induced infarct size.

The cytosolic enzymes CK-MB and LDH are sensitive markers used to diagnosis myocardial ischemia (28). When the cell is damaged, the cell membrane may be ruptured or become permeable, resulting in the leakage of CK-MB and LDH from the damaged cardiomyocytes to the bloodstream (29). The results of the present study detected a marked increase in CK-MB and $\mathrm{LDH}$ in the mouse model of myocardial ischemia. A previous study indicated that increases in serum CK-MB and LDH were associated with severe damage to myocardial tissue (30). Notably, the elevated levels of serum CK-MB and LDH in the model group were significantly reduced by pretreatment with DOE. These results indicated that DOE may attenuate damage to cardiomyocytes, reduce the leakage of cardiac markers and suppress the development of myocardial ischemia.

Free radicals are generated and accumulated during acute myocardial ischemia. MDA, which is a lipid peroxidation product, has been used to determinate the levels of oxygen free radicals mediated by myocardial injury (31). The present study demonstrated that DOE significantly reduced serum MDA levels, thus indicating that DOE may exert its protective effects against myocardial ischemia by reducing lipid peroxidation. SOD, which is an antioxidant enzyme, is beneficial in the scavenging of free radicals $(32,33)$. Pretreatment with $150 \mathrm{mg} / \mathrm{kg}$ DOE induced a significant increase in serum SOD activity, as compared with in the model group. These results indicated that DOE may affect endogenous antioxidants or oxidative stress. Previous studies reported that oxidative stress serves important roles in myocardial ischemia $(3,24)$; therefore, the antioxidant effects of DOE may prevent the deleterious effects of oxidative stress on the development of ischemia.

The protective effects of DOE against myocardial ischemia were further confirmed by histopathological examination. Basophilic components, including the nucleus and endoplasmic reticulum, are stained blue by hematoxylin, whereas eosinophilic-like proteins are stained pink by eosin (34). Mild cardiomyocyte necrosis and reduced inflammatory infiltration were detected in the DOE pretreatment groups, thus suggesting that DOE may alleviate cardiac infarction and decrease inflammatory cell recruitment. Apoptosis had been observed in cardiac pathologies, including hypoxia, ischemia reperfusion and myocardial ischemia (5). In the present study, TUNEL assays demonstrated that pretreatment with DOE decreased the degree of apoptosis in the heart. These data suggested that DOE may attenuate cardiac injury through inhibiting myocardial apoptosis.

Meis1 is a critical transcriptional regulator in cardiomyocyte proliferation, which may be considered a potential therapeutic target for heart regeneration (19). Stankunas et al examined cardiac development in mice lacking Meis1 and demonstrated that embryos without Meis1 displayed subcutaneous hemorrhage and died between embryonic day 14.5 and 15.5, confirming that Meis1 is essential for cardiac development (35). In the present study, the expression levels of Meis1 were decreased in the model group. However, this decrease was markedly ameliorated by pretreatment with DOE (75 and $150 \mathrm{mg} / \mathrm{kg}$ ); this may be a potential mechanism underlying the protective effects of DOE against myocardial ischemia. Therefore, Meis1 may be a novel therapeutic target for the development of anti-ischemia drugs. However, further studies are required to confirm this hypothesis.

In conclusion, the present study demonstrated that DOE possessed cardioprotective potential against LAD ligation-induced myocardial ischemia. The underlying mechanism is possibly associated with restoration of antioxidant enzyme activity, inhibition of apoptosis and upregulation of Meis1 expression. These findings may be beneficial for patients with myocardial ischemia; however, further studies are required to elucidate the precise molecular mechanisms underlying the effects of DOE.

\section{Acknowledgements}

The present study was supported by the Chongqing Science and Technology Commission (no. cstc2016jcyjA0296), the Fundamental Research Funds for the Central University (nos. XDJK2014B024 and XDJK2016E137), and the Innovative Project on Designing and Screening Drug Candidates of Chongqing (no. cstc2015zdcy-ztzx120003).

\section{References}

1. World Health Organization: Global status report on noncommunicable disease 2014. World Health Organization, Geneva, Switzerland, pp9-14, 2014. 
2. Gao E, Lei YH, Shang X, Huang ZM, Zuo L, Boucher M, Fan Q, Chuprun JK, Ma XL and Koch WJ: A novel and efficient model of coronary artery ligation and myocardial infarction in the mouse. Circ Res 107: 1445-1453, 2010.

3. Hori M and Nishida K: Oxidative stress and left ventricular remodelling after myocardial infarction. Cardiovasc Res 81: 457-464, 2009.

4. Sun J, Nguyen T, Aponte AM, Menazza S, Kohr MJ, Roth DM, Patel HH, Murphy E and Steenbergen C: Ischaemic preconditioning preferentially increases protein S-nitrosylation in subsarcolemmal mitochondria. Cardiovasc Res 106: 227-236, 2015.

5. Abbate A, Bussani R, Amin MS, Vetrovec GW and Baldi A: Acute myocardial infarction and heart failure: Role of apoptosis. Int J Biochem Cell Biol 38: 1834-1840, 2006.

6. Han SY, Li HX, Ma X, Zhang K, Ma ZZ and Tu PF: Protective effects of purified safflower extract on myocardial ischemia in vivo and in vitro. Phytomedicine 16: 694-702, 2009.

7. Ma X, Zhang K, Li H, Han S, Ma Z and Tu P: Extracts from Astragalus membranaceus limit myocardial cell death and improve cardiac function in a rat model of myocardial ischemia. J Ethnopharmacol 149: 720-728, 2013.

8. Ng TB, Liu J, Wong JH, Ye X, Wing Sze SC, Tong Y and Zhang KY: Review of research on Dendrobium, a prized folk medicine. Appl Microbiol Biotechnol 93: 1795-1803, 2012

9. Lin X, Shaw PC, Sze SC, Tong Y and Zhang Y: Dendrobium officinale polysaccharides ameliorate the abnormality of aquaporin 5, pro-inflammatory cytokines and inhibit apoptosis in the experimental Sjögren's syndrome mice. Int Immunopharmacol 11: 2025-2032, 2011.

10. Xia L, Liu X, Guo H, Zhang H, Zhu J and Ren F: Partial characterization and immunomodulatory activity of polysaccharides from the stem of Dendrobium officinale (Tiepishihu) in vitro. J Funct Foods 4: 294-301, 2012.

11. Wang Q, Gong Q, Wu Q and Shi J: Neuroprotective effects of Dendrobium alkaloids on rat cortical neurons injured by oxygen-glucose deprivation and reperfusion. Phytomedicine 17: 108-115, 2010.

12. Zhao X, Sun P, Qian Y and Suo H: D. candidum has in vitro anticancer effects in HCT-116 cancer cells and exerts in vivo anti-metastatic effects in mice. Nutr Res Pract 8: 487-493, 2014.

13. Lin X, Sze SCW, Tong Y, Zhang Z, Feng, Y, Chen JP, Ng TB, Lin X, Shaw PC and Zhang KY: Protective effect of Dendrobium officinale polysaccharides on experimental Sjögren's syndrome. J Complement Integr Med 7 (1), 2010.

14. Yang L, Liu SJ, Luo HR, Cui J, Zhou J, Wang XJ, Sheng J and $\mathrm{Hu}$ JM: Two new dendrocandins with neurite outgrowth-promoting activity from Dendrobium officinale. J Asian Nat Prod Res 17: 125-131, 2015

15. Chen CC, Wu LG, Ko FN and Teng CM: Antiplatelet aggregation principles of Dendrobium Loddigesii. J Nat Prod 57: 1271-1274, 1994.

16. Cui L, Li M, Feng F, Yang Y, Hang X, Cui J and Gao J: Meis1 functions as a potential AR negative regulator. Exp Cell Res 328: 58-68, 2014.

17. Yuan X and Braun T: An unexpected switch: Regulation of cardiomyocyte proliferation by the homeobox gene meis1. Circ Res 113: 245-248, 2013.

18. Azcoitia V, Aracil M, Martinez-A C and Torres M: The homeodomain protein Meis1 is essential for definitive hematopoiesis and vascular patterning in the mouse embryo. Dev Biol 280: 307-320, 2005.

19. Mahmoud AI, Kocabas F, Muralidhar SA, Kimura W, Koura AS, Thet S, Porrello ER and Sadek HA: Meis1 regulates postnatal cardiomyocyte cell cycle arrest. Nature 497: 249-253, 2013.
20. Ahn D, Cheng L, Moon C, Spurgeon H, Lakatta EG and Talan MI: Induction of myocardial infarcts of a predictable size and location by branch pattern probability-assisted coronary ligation in C57BL/6 mice. Am J Physiol Heart Circ Physiol 286: H1201-H1207, 2004.

21. Samsamshariat SA, Samsamshariat ZA and Movahed MR: A novel method for safe and accurate left anterior descending coronary artery ligation for research in rats. Cardiovasc Revasc Med 6: 121-123, 2005

22. Chu W, Qiao G, Bai Y, Pan Z, Li G, Piao X, Wu L, Lu Y and Yang B: Flavonoids from Chinese Viscum coloratum produce cytoprotective effects against ischemic myocardial injuries: Inhibitory effect of flavonoids on PAF-induced $\mathrm{Ca}^{2+}$ overload. Phytother Res 22: 134-137, 2008.

23. Zhao XY, Li GY, Liu Y, Chai LM, Chen JX, Zhang Y, Du ZM, Lu YJ and Yang BF: Resveratrol protects against arsenic trioxide-induced cardiotoxicity in vitro and in vivo. Br J Pharmacol 154: 105-113, 2008.

24. Plummer BN, Liu H, Wan X, Deschênes I and Laurita KR: Targeted antioxidant treatment decreases cardiac alternans associated with chronic myocardial infarction. Circ Arrhythm Electrophysiol 8: $165-173,2015$.

25. Queenthy SS and John B: Diosmin exhibits anti-hyperlipidemic effects in isoproterenol induced myocardial infarcted rats. Eur J Pharmacol 718: 213-218, 2013.

26. Gong LL, Fang LH, Wang SB, Sun JL, Qin HL, Li XX, Wang SB and Du GH: Coptisine exert cardioprotective effect through anti-oxidative and inhibition of RhoA/Rho kinase pathway on isoproterenol-induced myocardial infarction in rats. Atherosclerosis 222: 50-58, 2012.

27. Radhiga T, Rajamanickam C, Senthil S and Pugalendi KV: Effect of ursolic acid on cardiac marker enzymes, lipid profile and macroscopic enzyme mapping assay in isoproterenol-induced myocardial ischemic rats. Food Chem Toxicol 50: 3971-3977, 2012.

28. Derbali A, Mnafgui K, Affes M, Derbali F, Hajji R, Gharsallah N, Allouche N and El Feki A: Cardioprotective effect of linseed oil against isoproterenol-induced myocardial infarction in Wistar rats: A biochemical and electrocardiographic study. J Physiol Biochem 71: 281-288, 2015.

29. He H, Xu J, Xu Y, Zhang C, Wang H, He Y, Wang T and Yuan D: Cardioprotective effects of saponins from Panax japonicus on acute myocardial ischemia against oxidative stress-triggered damage and cardiac cell death in rats. J Ethnopharmacol 140: 73-82, 2012.

30. Quan W, Wu B, Bai Y, Zhang X, Yin J, Xi M, Guan Y, Shao Q, Chen Y, Wu Q and Wen A: Magnesium lithospermate $\mathrm{B}$ improves myocardial function and prevents simulated ischemia/reperfusion injury-induced $\mathrm{H} 9 \mathrm{c} 2$ cardiomyocytes apoptosis through Akt-dependent pathway. J Ethnopharmacol 151: 714-721, 2014.

31. Wu L, Qiao H, Li Y and Li L: Protective roles of puerarin and Danshensu on acute ischemic myocardial injury in rats. Phytomedicine 14: 652-658, 2007.

32. Qin F, Liu YX, Zhao HW, Huang X, Ren P and Zhu ZY: Chinese medicinal formula Guan-Xin-Er-Hao protects the heart against oxidative stress induced by acute ischemic myocardial injury in rats. Phytomedicine 16: 215-221, 2009.

33. Yu C, Fu F, Yu X, Han B and Zhu M: Cardioprotective effect of Ocotillol, a derivate of pseudoginsenoside F11, on myocardial injury induced by isoproterenol in rats. Arzneimittelforschung 57: 568-572, 2007.

34. Borst O, Ochmann C, Schönberger T, Jacoby C, Stellos K, Seizer P, Flögel U, Lang F and Gawaz M: Methods employed for induction and analysis of experimental myocardial infarction in mice. Cell Physiol Biochem 28: 1-12, 2011.

35. Stankunas K, Shang C, Twu KY, Kao SC, Jenkins NA, Copeland NG, Sanyal M, Selleri L, Cleary ML and Chang CP: Pbx/Meis deficiencies demonstrate multigenetic origins of congenital heart disease. Circ Res 103: 702-709, 2008. 